\title{
De la ingeniería Biomédica al aula de Matemáticas
}

\author{
Avenilde Romo-Vázquez \\ Centro de investigación en Ciencia Aplicada y Tecnología \\ Avanzada \\ Instituto Politécnico Nacional \\ avenilderv@yahoo.com.mx
}

Rebeca Romo-Vázquez

Centro Universitario de Ciencias Exactas e Ingenierías Universidad de Guadalajara, México rebeca.romo@cucei.udg.mx

Hugo Velez-Pérez

Centro Universitario de Ciencias Exactas e Ingenierías Universidad de Guadalajara, México hugo.velez@cucei.udg.mx,

Resumen: Con el objetivo de diseñar actividades didácticas de modelación lo más cercanas a un contexto "real", se realizó un trabajo colaborativo entre matemáticos educativos e ingenieros biomédicos. Este trabajo permitió conocer y analizar un contexto real de modelación matemática, el método de Separación Ciega de Fuentes (Blind Source Separation - BSS). La descripción del método de la BSS, que presentamos en la primera parte de esta comunicación, fue mostrada a profesores de matemáticas que cursaban la maestría en Matemática Educativa del CICATA-IPN y se les pidió generar actividades didácticas inspiradas en este contexto. En la segunda parte de 
esta comunicación, presentamos algunas de las actividades que ellos generaron y consideramos que son una buena base para el diseño de secuencias didácticas que puedan figurar en la formación matemática de futuros ingenieros.

Palabras clave: modelación matemática, contexto real, método BSS

\section{Introducción}

La modelización matemática se ha reconocido como una herramienta fundamental en la práctica de los ingenieros. En 1988 se publicó el estudio ICMI 3 realizado en el seno de la Comisión Internacional de la Enseñanza de las Matemáticas. El tema del estudio fue Matemáticas como disciplina de servicio, matemáticos, matemáticos educativos, investigadores y educadores, contribuyeron a este estudio. En su contribución, Pollak matemático que trabajó durante 35 años en los Laboratorios Bell y formó ingenieros en esta empresa, señala que en niveles técnicos avanzados los ingenieros necesitan en su práctica:

Antes que todo, necesitamos tener conocimiento del hecho que el pensamiento matemático, el pensamiento analítico, estructural, cuantitativo, sistemático, puede ser aplicado al mundo real y generar observaciones de gran valor; en otros términos que la modelización matemática es posible y puede ser eficaz. (Pollak, 1988, p.31)

La modelización es vista como el paradigma educativo que la formación matemática puede adoptar en lugar del clásico, teoría aplicaciones. Investigaciones que se han desarrollado para reconocer las matemáticas que figuran en las prácticas profesionales (Noss, Hoyles y Pozzi 2000: Kent y Noss

2001; Bissell y Dillon 2000; Bissell 2002 y 2004) analizados en detalle en (Romo-Vázquez, 2009) permiten caracterizar las matemáticas en las prácticas profesionales como: 
- Estas matemáticas se construyen en una relación estrecha con la práctica, en una comprensión a través del uso

- Sus dimensiones las más avanzadas tienden, cada vez más, a estar a cargo de especialistas o de programas computacionales

- Las necesidades de los no especialistas parecen desplazarse hacia la capacidad de manipular estas matemáticas como una herramienta de comunicación a través de los lenguajes específicos, esto contribuye a explicar por qué su rol es tan poco reconocido

Consideramos que para que la formación de futuros ingenieros pueda adaptarse a las necesidades matemáticas de la práctica, es necesario generar dispositivos didácticos donde la modelización matemática -construcción, adaptación y refinamiento de modelos- tenga un lugar central. Es por ello, que hemos desarrollado un proyecto conjunto, matemáticos educativos e ingenieros biomédicos para que el uso de modelos matemáticos en la práctica ingenieril sea base de actividades didácticas. En esta comunicación presentamos dos fases de este proyecto:

1. Análisis de modelos matemáticos en ingeniería biomédica y particularmente en el método de la BSS

2. Diseño de actividades didácticas por profesores de matemáticas considerando el contexto de la BSS

Presentamos primeramente el método de la BSS y posteriormente las actividades diseñadas por los profesores de matemáticas. 


\section{Contexto de la ingeniería biomédica: Separación Ciega de Fuentes}

El método de Separación Ciega de Fuentes es utilizado en ingeniería biomédica en diferentes aplicaciones. Nosotros analizamos el uso del método para mejorar el diagnóstico de la epilepsia.

\subsection{Descripción del Contexto que motiva el uso del método Separación Ciega de Fuentes}

En el área de la ingeniería biomédica, ingeniería al servicio de la salud, en el dominio de procesamiento de señales, más específicamente, se estudia la actividad eléctrica del organismo a través del registro de señales electrofisiológicas. Las señales registradas permiten, en un trabajo de clínica, determinar el estado de salud de las personas y diagnosticar la presencia de una posible patología. Por lo que es muy importante que este registro sea lo más fiel posible a la actividad eléctrica de los órganos. Es decir, durante el registro existen diferentes interferencias y/o perturbaciones que alteran el registro de las señales y por consecuencia su interpretación. Algunos ejemplos de señales electrofisiológicas son: el electrocardiograma (ECG), el electroencefalograma (EEG), el electromiograma (EMG), etc. Por ejemplo, el electroencefalograma (EEG) es el método más usado para registrar la actividad eléctrica del cerebro, mediante electrodos que se colocan sobre el cuero cabelludo. Estos electrodos se colocan siguiendo estándares internacionales, por ejemplo el sistema 10 - 20 (ver figura 1). Si bien, estos electrodos registran la actividad eléctrica cerebral, también registran otro tipo de actividad, como puede ser actividad electrofisiológica que no sea de origen cerebral (i.e. actividad ocular, actividad muscular, actividad cardiaca), así como ruido. Tanto al ruido como a la actividad no cerebral que se registra se les conoce como perturbaciones. 

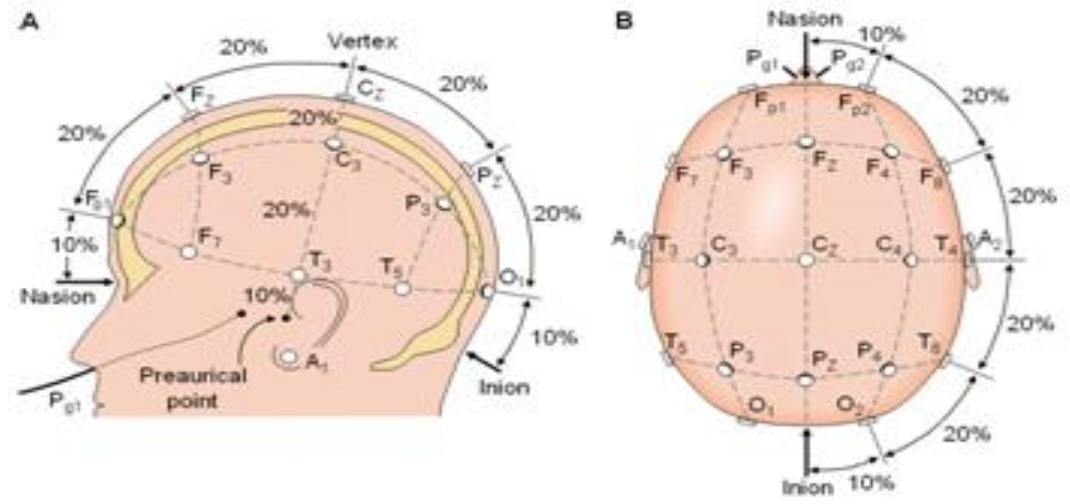

Figura 1. Sistema internacional 10 - 20 para la colocación de electrodos

Con la finalidad de tener registros exentos de perturbaciones se han creado métodos de tratamiento de señal como la separación ciega de fuentes (BSS), el cual consiste en separar a partir de los registros de EEG las fuentes (señales) que tienen un origen cerebral y las fuentes que no tienen un origen cerebral. Una vez que las señales de EEG son separadas en sus fuentes de origen se procede a identificar las de origen cerebral y las de origen no cerebral (extra - cerebrales). Posteriormente las fuentes clasificadas como extra cerebrales son eliminadas y se conservan únicamente las fuentes cerebrales.

Esta descripción fue hecha por ingenieros biomédicos en respuesta a nuestra demanda, la cual nos permite conocer el contexto que motiva/requiere el uso del método BSS. Del trabajo colaborativo entre ingenieros biomédicos y matemáticos educativos resulta una descripción más detallada del método. Lo anterior es realizado para transponer/adecuar dicho contexto en un contexto extra-matemático para el diseño de actividades didácticas. 


\subsection{Método de la BSS y su viabilidad como contexto de actividades didácticas de modelación}

Como se mencionó anteriormente, la idea de la BSS consiste en encontrar las fuentes de origen a partir únicamente del conocimiento de una mezcla de señales desconocidas (las observaciones). Esta mezcla es el resultado de la combinación de diversas fuentes de origen a través de una matriz de mezcla también desconocida. Teniendo únicamente como conocimiento el resultado de la mezcla (observaciones). A fin de encontrar las fuentes, el objetivo de la BSS es encontrar la matriz inversa (a la matriz de mezcla), esto sin tener ningún conocimiento previo, ni de las fuentes ni del sistema de mezcla. En el caso de un registro de EEG, consideramos el EEG como el resultado de una mezcla de fuentes cerebrales y de fuentes extra cerebrales. Los registros de EEG son el resultado de la combinación de diversas fuentes s (cerebrales y extra cerebrales), esta combinación es registrada por los electrodos colocados sobre el cuero cabelludo (las observaciones) x (Figura, 2).

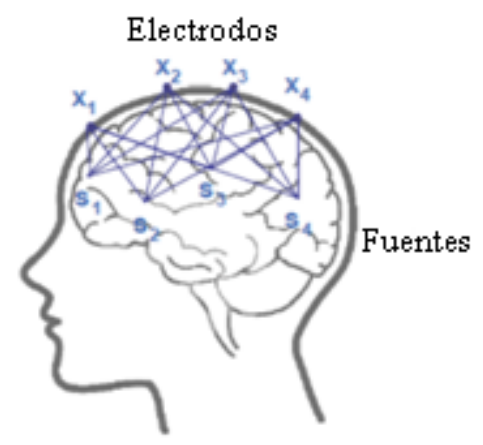

Figura 2. Combinación entre electrodos y fuentes.

El principio de la solución de la BSS está basado en el álgebra lineal, en el uso de matrices y operaciones sobre éstas como la multiplicación que nos permite resolver el problema inverso presentado. Es decir que a partir de una matriz de 
señales desconocidas $\mathbf{x}$ debemos resolver el problema inverso y encontrar las matrices que multiplicadas (s y A) dieron como resultado la mezcla $\mathbf{x}$.

Desde el punto de vista formal, la separación ciega de fuentes consiste en estimar $P$ señales desconocidas $\mathbf{s}$ (las fuentes) a partir del conocimiento únicamente de $Q$ mezclas de las señales $\mathbf{x}$ (las observaciones). El término ciego significa que tanto las fuentes $\mathbf{s}$ como el sistema de mezcla $\mathbf{A}$ son

desconocidos

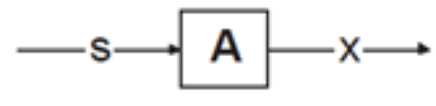

\subsubsection{Modelo de la BSS}

El modelo espacial de la mezcla al instante k está definido por (caso ideal, sin ruido):

$x(k)=A s(k)$

donde:

$$
x(k)=\left[x_{1}(k), \ldots, x_{Q}(k)\right]^{T}
$$

es el vector de señales observadas (electrodos)

$$
s(k)=\left[s_{1}(k), \ldots, s_{p}(k)\right]^{T}
$$

es el vector de las fuentes de origen (desconocido)

$\boldsymbol{A}(\boldsymbol{Q} \times \boldsymbol{P})$ la matriz de mezcla (desconocida). 
El objetivo de la BSS es la estimación de una matriz de separación B, que permita la estimación de las fuentes de origen s a partir de las señales medidas $\mathrm{x}$ :

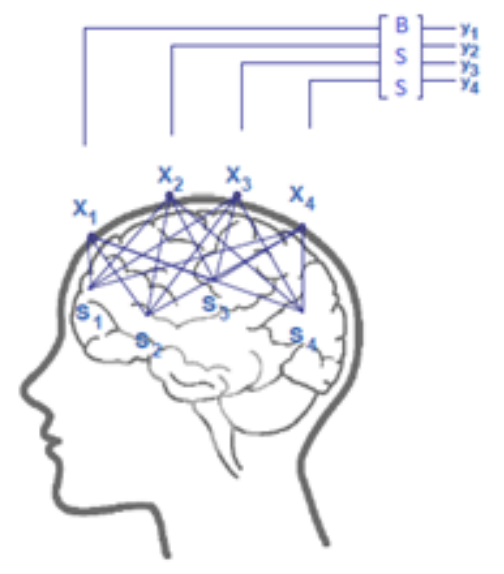

Figura 3. Principio del método de la BSS

Aquí vemos aparecer la motivación del método y ésta no es matemática sino contextual, se desea conocer las fuentes $s$ a partir de las observaciones $x$ obtenidas. La explicación hecha del método BSS, muestra que este método es el óptimo para poder lograr este objetivo.

Para fines clínicos el interés está en conocer las fuentes de origen cerebral, sin embargo el registro sólo muestra la mezcla de las diversas fuentes. Sin ningún conocimiento a priori de las fuentes cerebrales, solucionar este problema resulta una tarea muy complicada a resolver. Los resultados obtenidos con el uso de la BSS en clínica son prometedores. 


\section{Actividades didácticas propuestas por profesores de matemáticas}

La descripción del método de la BSS fue propuesto en un curso de maestría de Matemática Educativa en el Programa de Matemática Educativa de Cicata-IPN, en la modalidad en línea y a distancia. Los estudiantes del curso eran profesores de matemáticas de diferentes partes de Latinoamérica.

En el curso, además del método se les propuso una metodología para el diseño de actividades didácticas basadas en modelación (Macias, 2012). Con estos dos documentos los profesores del curso fueron solicitados a generar actividades didácticas de modelación considerando el método de la BSS como una aplicación real de la ingeniería, que podía convertirse/transformarse en una actividad didáctica de modelación. Para realizar esta actividad, los profesores participaron en un foro de dudas que era atendido tanto por matemáticos educativos e ingenieros biomédicos. A partir del foro pudimos observar que antes del diseño de la actividad didáctica, los profesores requieren comprender el método de la BSS y en efecto lo transponen (lo modifican para volverlo comprensible).

Para ilustrar lo anterior consideramos la siguiente imagen puesta en el foro por uno de los participantes que llamaremos el profesor Luis:

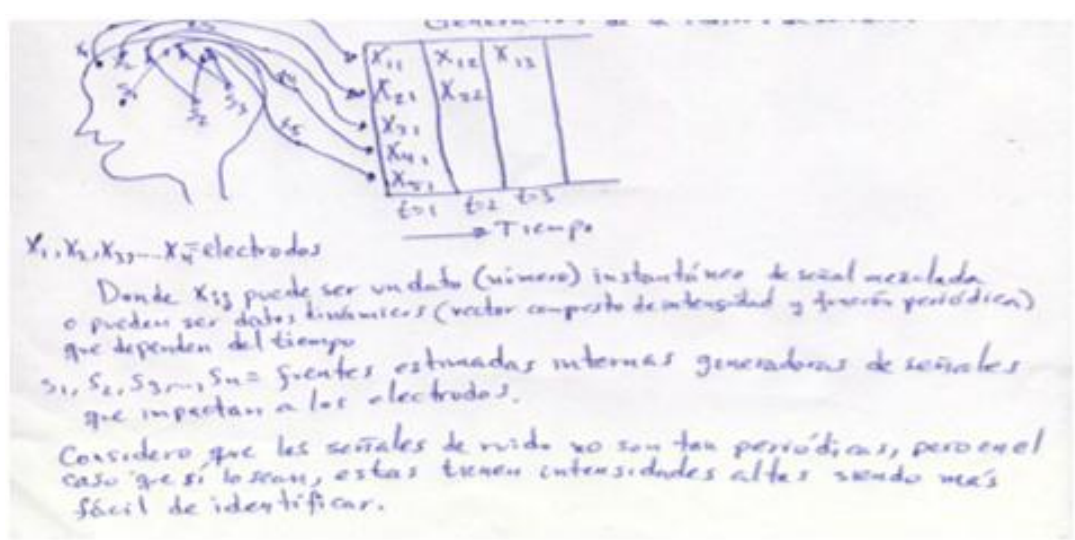

Figura 4. Documento expuesto en el foro por el profesor Luis 
En este documento producido por el profesor Luis se muestra un dibujo en el que se ilustran los electrodos como generadores de datos, $x_{1}, x_{2}, x_{3} \ldots x_{n}$ en relación al tiempo. Es interesante notar como en este dibujo los datos van de los electrodos hacia una tabla, las columnas aparecen valores distintos para t (tiempo). El profesor especifica que las $x_{i j}$ pueden ser datos numéricos producidos al instante de una señal mezclada (lenguaje del contexto) en relación a un tiempo, o bien "pueden ser datos dinámicos (vector de intensidad y función periódica) que dependen del tiempo". Aquí reconoce al vector como el elemento matemático que permite modelar la información obtenida por los electrodos. Al final de su documento escribe: "Considero que las señales de ruido no son tan periódicas, pero en el que caso que lo sean, éstas tienen intensidades altas siendo más fácil de identificar." Lo anterior nos hace suponer que el profesor Luis intenta reconocer la naturaleza matemática (a través de la periodicidad) de las señales de ruido. El lenguaje privilegiado es el del contexto, no habla de funciones sino de señales y asocia la intensidad al elemento que permite identificar el tipo de señal, asociándolo a lo periódico.

En este mismo foro otro de los profesores que llamaremos profesor Diego, reconoce, a partir de un análisis sobre el documento dado (descripción de la BSS) y otros artículos producto de su propia investigación, el principio de la BSS y lo considera para el caso de ecuaciones polinómicas:

De acuerdo a lo leído y analizado, en el escrito, y en otros artículos, puedo formular lo siguiente: No conocemos las fuentes (s) ni la matriz de mezcla $(A)$, sólo se conoce $(x) . x=A . s$ De acuerdo a lo anterior podría decir lo siguiente: $x^{3}+x^{2}-3 x+1$, es una ecuación polinómica, que ha sido, perturbada por otra, con características lineales. Cuál será la función original polinómica, y cuál la perturbadora. ¿Podría ser este un contexto, que hace relación a la separación ciega de fuentes? (Participación del profesor Diego en el foro del curso) 
La profesora Adriana al igual que el profesor Diego señala que ha investigado sobre la BSS encontrando que existen algoritmos muy complejos para encontrar las fuentes originales:

El objetivo de esta actividad es diseñar una actividad de modelación donde podamos aplicar la teoría BSS. Yo estoy pensando en adaptar esta teoría de señales, simplificándola para poder trabajar con estudiantes de secundaria (bachillerato), he podido constatar que existen algoritmos bastante complejos para resolver la ecuación matricial original sin conocer las señales de origen ni la matriz mezcla. Por lo tanto voy a intentar diseñar una actividad dónde a partir de determinados datos los alumnos puedan construir la matriz mezcla además de contar con los datos de las señales que se recepcionan. Estuve leyendo el problema de la "fiesta de cóctel", se me complica en pensar ¿cómo medir las señales originales? para darle sentido a una actividad de clase. Es difícil encontrar actividades que expliquen cómo se aplica esta teoría en un nivel básico. (Participación de la profesora Adriana en el foro del curso)

La profesora Adriana explicita primeramente la consigna de la actividad que les fue pedida e inmediatamente señala la necesidad de adaptar la BSS (vista como teoría) al nivel en el que ella labora, el bachillerato. Manifiesta que ha investigado sobre la BSS al confirmar que existen algoritmos "bastante complejos para resolver la ecuación matricial original sin conocer las señales de origen ni la matriz mezcla". En este extracto Adriana enuncia el principio del método, lo que permite hacer y es éste el que debe considerar para el aula, para lo cual señala deberá tener datos de las señales que se captan. Asimismo, Adriana menciona que en sus investigaciones ha encontrado problemas basados en el método como este de la "fiesta de cóctel" pero parece todavía no serle útil para diseñar su propia actividad. 
Lo anterior, nos permite ilustrar esta primera etapa en el diseño que consiste en apropiarse del contexto para luego adaptarlo al aula. Y más precisamente a tareas que evocan el contexto real de aplicación pero que puedan ser realizadas en una clase de matemáticas. Analizamos a continuación la actividad propuesta por Adriana par la cual considera y presenta la siguiente tabla:

Algo sobre escalas musicales:

Tabla de frecuencias de las notas musicales de la escala cromática:

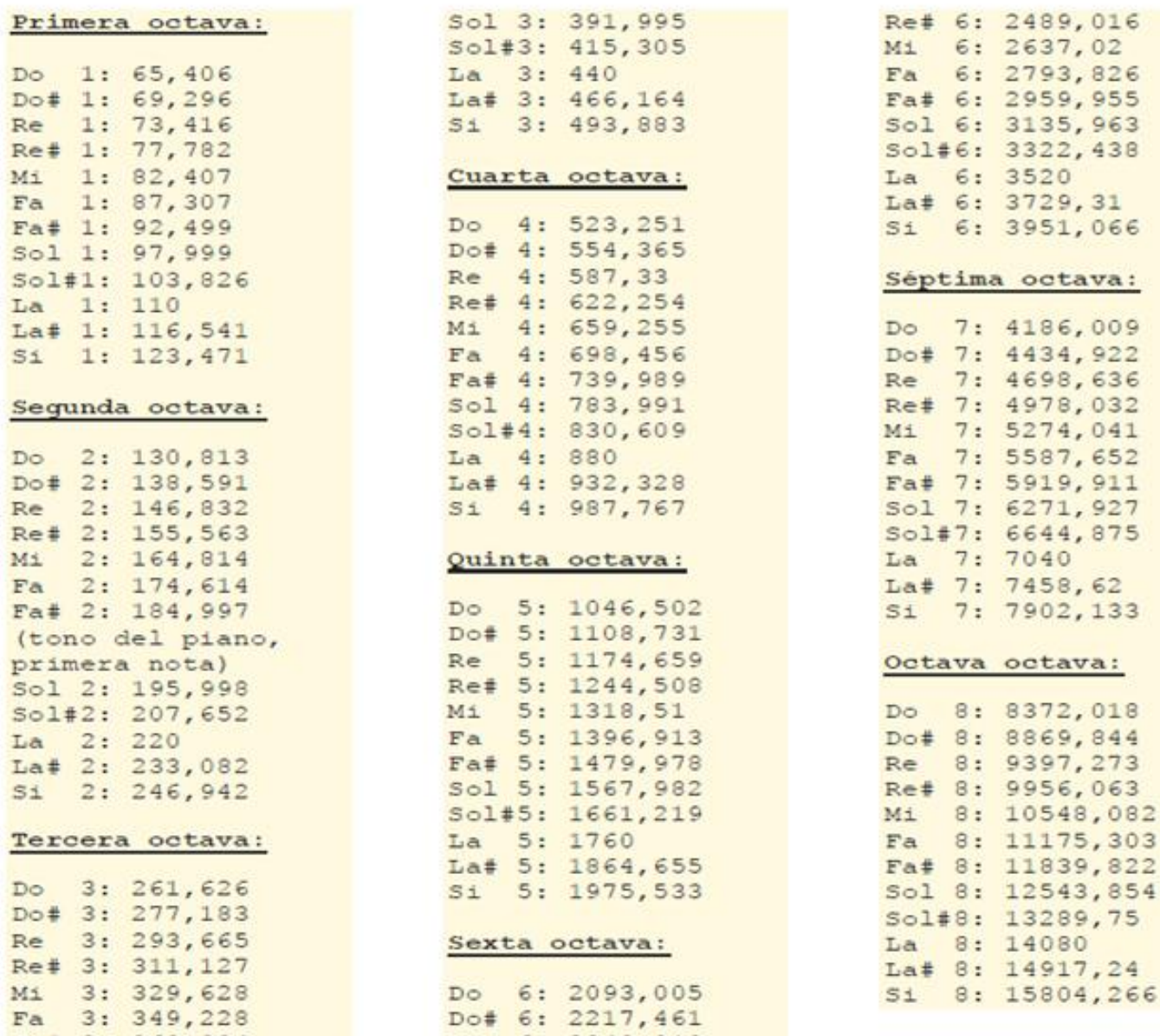

Figura 5. Tabla de frecuencias de las notas musicales de la escala cromática 
La tabla de frecuencias aparece acompañada de las siguiente explicación que muestra cómo leer la tabla:

El tono del piano (primera nota) corresponde a un Fa\# 3 (tercera octava) cuya frecuencia corresponde a 184,997 HZ, un piano normalmente tiene unas 72 teclas, por lo tanto tiene 6 octavas. El tono del violín corresponde a un SI 4 (nota Si de la cuarta octava) cuya frecuencia corresponde a 987,767 HZ y tiene 3 octavas.

A pesar de que ella escribe 184,997 HZ que corresponde al valor de la segunda escala y no al de la tercera que es: 369,994 HZ.

\section{El problema}

En un estudio de grabación se realizan pruebas de audio de un dúo musical formado por un piano y un violín. Se ubican en la sala de grabación dos micrófonos para grabar la ejecución de la pieza instrumental, estos micrófonos están conectados a una consola que graba el tema que ambos instrumentos están ejecutando, el ingeniero de sonido se percata que la consola está distorsionando el sonido original, las frecuencias de las notas ejecutadas por los instrumentos musicales no son las que quedan registradas en la grabación. El ingeniero registra las mediciones de frecuencias en un instante dado que obtiene con el micrófono 1 (M1) y con el micrófono 2 (M2) y descubre que las distorsiones generadas en la grabación están dadas según los datos de la matriz

$$
A=\left(\begin{array}{cc}
-4 & 2 \\
5 & 3
\end{array}\right)
$$

(dónde los datos de la fila 1 corresponden a las distorsiones de los registros captados en el M1 y los de la fila 2 a las distorsiones de las frecuencias captadas por M2, la columna 1 son las distorsiones que sufre la frecuencia del piano y los datos de la columna 2 las que sufre la frecuencia del violín). Los registros de frecuencia obtenidos de la grabación en ese instante dado (seg.) 
son: $10760 \mathrm{~Hz}$ (en el M1) y $25270 \mathrm{~Hz}$ (con el M2). En ese instante de registro, ¿cuáles son las notas reales ejecutadas por los instrumentos y en qué octava?

Podemos ver cómo Adriana a partir de la consideración de la tabla de frecuencias de las notas musicales de la escala cromática logra generar un problema que puede ser llevado al aula. Notemos que este problema está en una etapa intermedia de diseño, pues faltan datos, por ejemplo del tiempo; no se explicitan las tareas que deberá resolver el estudiante o la forma en que le será presentado; en qué tema del programa será presentado, etc. Sin embargo el contexto de la BSS ha sido adaptado a través de este de las frecuencias de la escala cromática y el primer desglose en términos de praxeologías mixtas (tarea no matemática, técnica matemática, tecnologías matemáticas y no matemáticas, teoría) es presentado como se muestra a continuación:

\section{Tareas no matemáticas:}

T1: Determinar nota y octava que ejecuta el piano en el instante registrado.

T2: Determinar nota y octava que ejecuta el violín en el instante registrado.

\section{Técnica matemática:}

Ô1: Resolución de la ecuación matricial:

$$
\left(\begin{array}{l}
10760 \\
25270
\end{array}\right)=\left(\begin{array}{cc}
-4 & 2 \\
5 & 3
\end{array}\right)\left(\begin{array}{l}
P \\
V
\end{array}\right)
$$

\section{Tecnologías matemáticas:}

Existencia y unicidad de la inversa de una matriz cuadrado no singular (determinante no nulo).

Definición y propiedades de la multiplicación de matrices.

Notemos que esta idea de praxeología mixta propuesta en la metodología para el diseño incluye este componente de tecnología no matemática, validaciones, explicaciones y justificaciones que provienen del contexto no matemático considerado. Como mencionamos en la introducción de esta comunicación las actividades propuestas por los profesores e ilustradas aquí a partir de la 
propuesta por Adriana, constituyen una base para el desarrollo de secuencia didácticas que puedan ser llevadas al aula.

\section{Conclusión}

El diseño de actividades didácticas basadas en modelación solicita un trabajo arduo para elegir y adaptar contextos extra-matemáticos 0 modelos matemáticos en uso. La elección del contexto extra-matemático y sobre todo su adaptación requiere un trabajo colaborativo con los expertos en dicho contexto. En este caso los ingenieros biomédicos colaboraron tanto en la fase de descripción de los modelos matemáticos utilizados en su contexto y en el curso de Matemática Educativa, particularmente en el foro. El trabajo con profesores de matemáticas es una fase fundamental y solicita:

- una primera transposición del contexto (descripción hecha del contexto)

- un marco institucional propicio

- una metodología para el diseño

El trabajo realizado por los profesores muestra que una segunda trasposición se ejerce antes del diseño de las actividades, ésta resulta necesaria para apropiarse del contexto extra-matemático y poderlo adaptar al aula. El diseño de las actividades está dado en términos de praxeologías mixtas (tareas, técnicas, tecnologías y teoría), lo cual es sugerido por la metodología propuesta y permite generar un "primer diseño didáctico"

Todas las actividades propuestas requieren de un segundo y hasta tercer diseño para poder ser probadas con estudiantes. Lo anterior es desarrollado en el marco de tesis de maestría y ha mostrado que las actividades propuestas por los profesores son una base para poder diseñar secuencias más elaboradas. El trabajo aquí presentado, a pesar de estar en una primera fase, nos parece ser una vía para acercar la modelación matemática que tiene lugar 
en escenarios reales a la formación matemática, particularmente de futuros ingenieros.

1 El registro de la actividad cerebral y no cerebral de manera conjunta es inevitable, debido a que los pelectrodos se colocan sobre el cureo cabelludo y registral a la vez otro tipo de actividad electrofisiológica como la actividad cardiaca, la actividad muscular y la actividad ocular, entre las más comunes.

\section{Referencias}

Bissell, C.C. (2000). Telling tales: models, stories and meanings. For the learning of mathematics, 20(3), 3-11.

Bissell, C.C. (2002). Histoires, héritages et herméneutique : la vie quotidienne des mathématiques de l'ingénieur. Annales des Ponts et Chaussées, 107-8, 4-9.

Chevallard, Y. (1999). La recherche en didactique et la formation des professeurs : problématiques, electroencephalogram by approximate joint diagonalization of second order statistics. Clinical Neurophysiology, 119(12). 2677-2686.

Jung, T., Makeig, S., Humphries, C., Lee, T., Mckeown, M., Iragui, V. \& Sejnowski, T. (2000). Removing e lectroencephalographic artifacts by blind source separation. Psychophysiology, 37(02). 163-178.

Howson, G., Kahane, J. P., Lauginie, P., Turckheim E. (1988). Mathematics as a Service Subjec. Cambridge: Cambridge University Press (ICMI Study Series).

Kachenoura, A. (2006). Traitement Aveugle de Signaux Biomédicaux. Traitement du signal et telecommunications, L'Universite de Rennes 1, France.

Kent, P. \& Noss, R. (2002). The mathematical components of engineering expertise: The relationship between doing and understanding mathematics. Proceedings of the IEE Second Annual Symposium on Engineering Education: Professional Engineering Scenarios 2, 39/1-39/7, London UK.

Macias (2012). Uso de las nuevas tecnologías en la formación matemática de ingenieros. Tesis de maestria. CICATA, unidad Legaria -IPN

Noss, R; Hoyles, C; Pozzi, S. (2000). Working Knowledge: Mathematics in use, In A. Bessot \& J. Ridgway (Eds.), Education for Mathematics in the workplace, (pp.17-35). Dordrecht: Kluwer Academic Publishers. 
Romo Vázquez, A. (2009). Les mathématiques dans la formation d'ingénieurs. Paris: Irem de Paris.

R. Romo-Vazquez, H. Velez-Perez, R. Ranta, V. Louis-Dorr, D. Maquin, L. Maillard. (2012). Blind source separation, wavelet denoising and discriminant analysis for EEG artefacts and noise cancelling, Biomedical Signal Processing and Control, 7(4). 389-400. 


\section{Notas biográficas}

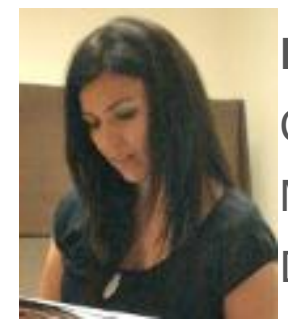

Dr. Avenilde Romo Vázquez Recibió el grado de Doctora en Ciencias en Didáctica de las Disciplinas, en la especialidad de Matemáticas, en la Universidad Paris 7en París, Francia. Desarrolla su investigación en la formación matemática de futuros ingenieros y en el rol de la modelación matemática.

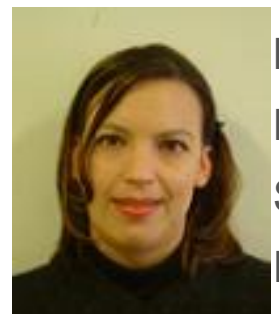

Dra. Rebeca del Carmen Romo Vázquez Recibió el grado de Doctora en Ciencias con la especialidad en Ingeniería por la Salud, en el Institut National Polytechnique de Lorraine en Nancy, Francia. Sus intereses son en tratamiento de señales de origen biológico.

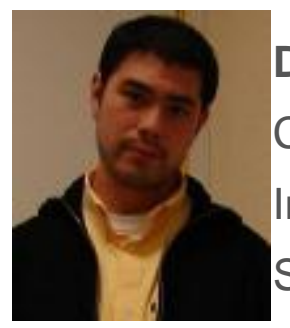

Dr. Hugo Abraham Vélez Pérez Recibió el grado de Doctor en Ciencias con la especialidad en Ingeniería por la Salud, en el Institut National Polytechnique de Lorraine en Nancy, Francia. Sus intereses son en tratamiento de señales de origen biológico.

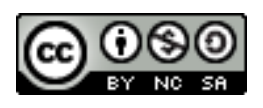

Esta obra está bajo una licencia de Creative Commons Reconocimiento-NoComercial-Compartirlgual 2.5 Mexico. 